\title{
Obtusifolin inhibits high glucose-induced mitochondrial apoptosis in human umbilical vein endothelial cells
}

\author{
YU TANG $^{1}$, ZHI-YING ZHONG ${ }^{2}$, YAN-FENG LIU ${ }^{1}$ and GOU-TAI SHENG ${ }^{1}$ \\ ${ }^{1}$ Department of Cardiology, People's Hospital of Jiangxi, Nanchang, Jiangxi 330006; ${ }^{2}$ Department of \\ Cardiology, The Fourth Affiliated Hospital of Nanchang University, Nanchang, Jiangxi 330003, P.R. China
}

Received June 28, 2017; Accepted September 28, 2017

DOI: $10.3892 / \mathrm{mmr} .2018 .9251$

\begin{abstract}
DM is often accompanied by macrovascular complications. Obtusifolin, which is an anthraquinone-based compound with antioxidant activity, is obtained from the seeds of Cassia obtusifolia. In this study, the potential effect of obtusifolin was investigated in human umbilical vein endothelial cells. The results from flow cytometry analysis revealed that pretreatment with obtusifolin depressed the production of cellular reactive oxygen species that was induced by high glucose content. Moreover, the results showed that pretreatment with obtusifolin reduced the level of malondialdehyde, as well as recovered the activities of mitochondrial complex I/III, catalase and superoxide dismutase. Furthermore, flow cytometry analysis also revealed that mitochondrial membrane potential and cell apoptosis were recovered, and inhibited by obtusifolin, respectively. The expression of X chromosome-linked IAP was upregulated, whereas the expressions of poly ADP-ribose polymerase and cysteinyl aspartate specific proteinase-3/9 were downregulated by the pretreatment with obtusifolin. Notably, the western blot analyses showed that the release of Omi/HtrA2 into the cytosol was prevented by the pretreatment with obtusifolin. Conclusively, it was suggested that obtusifolin may provide protection against mitochondrial apoptosis largely through inhibition of the release of Omi/HtrA2 from mitochondria into cytosol.
\end{abstract}

\section{Introduction}

Diabetes mellitus (DM) can lead to various vascular complications, and thus, cause cardiovascular dysfunction, which is often coupled with high morbidity and mortality rates. It has been reported that hyperglycemia plays an essential role in the pathogenesis of diabetic vascular complications $(1,2)$.

Correspondence to: Dr Zhi-Ying Zhong, Department of Cardiology, The Fourth Affiliated Hospital of Nanchang University, 133 Plaza Road, Nanchang, Jiangxi 330003, P.R. China

E-mail: zhiyingzhong112@163.com

Key words: obtusifolin, reactive oxygen species, mitochondrial apoptosis, Omi/HtrA2, human umbilical vein endothelial cells
Apoptosis is a prominent phenotype in the hyperglycemia injury model, which affects the function of vascular endothelium during tissue damage $(3,4)$. Vascular endothelial dysfunction is recognized as the main pathophysiological basis of diabetic angiopathy. Hyperglycemia-induced oxidative stress, an important feature of diabetes, is one of the major consequences of diabetes that may lead to production of reactive oxygen species (ROS) and may also cause mitochondrial dysfunction (5). Additionally, high glucose (HG) levels can induce apoptotic response in endothelial cells, thereby leading to endothelial injury (6). Numerous evidences indicate a link between ROS generation and high glucose-induced apoptosis in endothelial cells $(7,8)$. Production of ROS is a part of the mitochondrial respiratory chain, which can cause oxidative damage and aging (9). During the progress of diabetes mellitus, excessive ROS production induces the loss of mitochondrial membrane potential (MMP) along with an increase in mitochondrial membrane permeabilization (10). Subsequently, amounts of important proteases or proteins that are directly related to apoptosis are released from the mitochondria into the cytosol, which initiates the apoptotic cascade and accelerates apoptosis $(11,12)$. The protein, $\mathrm{Omi} / \mathrm{HtrA} 2$, is recognized as a pro-apoptotic serine protease, which is located in the mitochondrial inter-membrane space. It is reported that $\mathrm{Omi} / \mathrm{HtrA} 2$ promotes cell apoptosis mainly through the caspase-dependent pathways and caspase-independent pathways. In the former pathway, Omi/HtrA 2 is transferred into the cytoplasm. After wards, Omi/HtrA2 activates the caspase cascade by binding to the IAPs (inhibitor of apoptosis proteins), which finally induces cell apoptosis. The latter relies on the activity of protease itself to cause apoptosis $(13,14)$.

Recently, the use of traditional Chinese medicine or combined therapy of Chinese and Western medicine in the treatment of diabetes has increasingly attracted attention. Obtusifolin is an anthraquinone compound obtained from the seeds of Cassia obtusifolia, which is a traditional Chinese medicine. It is reported that obtusifolin can lower blood pressure and reduce serum cholesterol levels. Meanwhile, its anti-oxidant, anti-fungal, and neuroprotective activities have also been reported (15-17). However, the potential effects of obtusifolin on high-glucose-induced injury have not yet been clearly illustrated and the underlying mechanisms remain obscure. 
Our results have demonstrated that obtusifolin can protect human umbilical vein endothelial cells (HUVECs) from oxidative stress and mitochondrial apoptosis, which may otherwise be induced by high glucose treatment. The protective effect may be attributed to the inhibition of the release of Omi/HtrA2 into the cytosol. Our research would provide molecular clues to provide basis for further theoretical research and will be an evidence for the potential of obtusifolin to be a potent agent in the treatment of diabetes mellitus.

\section{Materials and methods}

Cell culture. HUVECs were obtained from the American Type Culture Collection (ATCC; Manassas, VA, USA). Cells were cultured in low-glucose Dulbecco's modified Eagle's medium (DMEM; Invitrogen Life Technologies, Carlsbad, CA, USA) that was supplemented with $10 \%$ fetal bovine serum, $100 \mathrm{U} / \mathrm{ml}$ penicillin and $100 \mu \mathrm{g} / \mathrm{ml}$ streptomycin (Sigma-Aldrich; Merck KGaA, Darmstadt, Germany). The cells were then incubated in a humidified atmosphere with $5 \% \mathrm{CO}_{2}$ at $37^{\circ} \mathrm{C}$.

Cell treatment. Six treatment groups were designed for the subsequent experiments: i) Control group (control), where cells were incubated with $5.5 \mathrm{mM}$ glucose; ii) mannitol group, where cells were treated with $33 \mathrm{mM}$ mannitol for $48 \mathrm{~h}$; iii) high glucose group (H-Glu), where cells were incubated with $33 \mathrm{mM}$ glucose for $48 \mathrm{~h}$; iv) obtusifolin pretreatment groups, where cells were preserved in the medium containing obtusifolin (Solarbio, Beijing, China) at different doses (5, 7.5 and $10 \mathrm{mg} / \mathrm{ml}$ ) for $6 \mathrm{~h}$ prior to the treatment with $33 \mathrm{mM}$ glucose for $48 \mathrm{~h}(\mathrm{H}-\mathrm{Glu}+5, \mathrm{H}-\mathrm{Glu}+7.5, \mathrm{H}-\mathrm{Glu}+10)$. After incubation, HUVECs were harvested for subsequent experiments.

Cell viability assay. For the cell viability assay, $1 \times 10^{4} \mathrm{HUVECs}$ were seeded in a 96-well plate. After being serum-starved overnight, the cells were treated with glucose $(11-33 \mathrm{mM})$ for 12,24 , and 48 h, separately. Following this, CCK-8 solution was added to the 96 -well plate. The plate was incubated in a $\mathrm{CO}_{2}$ incubator at $37^{\circ} \mathrm{C}$ for $4 \mathrm{~h}$ and then measured according to the protocol provided by the manufacturers of the CCK-8 detection kit (Cwibo, China).

Flow cytometer analysis for cellular ROS. The HUVECs were treated as above. Total and mitochondrial-specific ROS production was measured using CM-H2DCFDA probe (Invitrogen Life Technologies) and mitoSOX dye (Molecular Probes, Eugene, OR, USA), respectively. Briefly, cells were harvested and rinsed in pre-warmed phosphate-buffered solution (PBS) containing CM-H2DCFDA or mitoSOX in the dark for $20 \mathrm{~min}$ at $37^{\circ} \mathrm{C}$. The levels of ROS were determined using a BD FACSCalibur flow cytometer (Becton-Dickinson, San Jose, CA, USA) according to the manufacturer's instructions.

Flow cytometry analysis for MMP. The MMP in HUVECs was determined following the JC-1 dye staining method (Beyotime Institute of Biotechnology, Shanghai, China). The cells seeded in 6-well plates were treated as above and stained using the fluorescent dye, JC-1, at $37^{\circ} \mathrm{C}$ for $20 \mathrm{~min}$ in the dark. The cells were resuspended in PBS and analyzed using flow cytomety (Becton-Dickinson). The results then were calculated as previously described (18) and expressed as the proportion of cells with a low MMP.

Flow cytometry analysis for apoptosis. A fluorescein isothiocyanate (FITC)-Annexin V apoptosis detection kit (Invitrogen Life Technologies) was used to quantify the numbers of apoptotic cells. Cells in the 96-well plate (at a density of $1 \times 10^{4}$ cells/ well) were treated as instructed by the manufacturer's instructions and then washed with PBS. They were then stained with Annexin V and PI for $20 \mathrm{~min}$ at room temperature. The level of apoptosis was detected by measuring the fluorescence of cells using flow cytometer (Becton-Dickinson).

Immunofluorescence assay. Firstly, HUVECs were fixed using methanol. To block unspecific antigens, cells were incubated with $5 \%$ fetal bovine serum (FBS), $0.1 \%$ Triton X-100 in $1 \mathrm{X}$ PBS buffer. Then, HUVECs were incubated with anti-factor VIII antibody (Abcam, Cambridge, UK) at $4^{\circ} \mathrm{C}$ overnight after washing with $1 \mathrm{X}$ PBS thrice. After removal of the antibody solution, HUVECs were incubated with secondary FITC-conjugated anti-mouse antibody (Sigma-Aldrich; Merck $\mathrm{KGaA}$ ) at $37^{\circ} \mathrm{C}$ for $30 \mathrm{~min}$, and their nuclei were stained with DAPI for $15 \mathrm{~min}$. The HUVECs were then mounted on a slide and observed under a fluorescence microscope (Olympus, Tokyo, Japan).

Antioxidant enzyme activity assays and malondialdehyde levels. The HUVECs seeded in a 6-well plate (at a density of $1 \times 10^{5}$ cells/well) were treated as described above. According to the manufacturer's instructions, the activity of superoxide dismutase (SOD) and catalase (CAT) was measured using the SOD assay kit (Beyotime Institute of Biotechnology) and the CAT assay kit (Solarbio), respectively. Malondialdehyde (MDA) levels were detected using a malondialdehyde assay kit (Solarbio).

Mitochondrial respiratory chain activities assays. HUVECs $\left(1 \times 10^{5}\right)$ were seeded and treated as described above. The enzyme activities of complex I (NADH dehydrogenase) and complex III (cytochrome $b c 1$ complex) were examined using spectrophotometric method as previously described (19). Briefly, cells were lysed with $100 \mathrm{mM}$ Tris- $\mathrm{HCl}$ (pH 7.4) buffer containing $250 \mathrm{mM}$ sucrose and $2 \mathrm{mM}$ EDTA. The activity of complexes I and III was detected by measuring the reduction of 2,6-dichlorophenolindophenol (DCPIP) at 520-600 nm (extinction coefficient, $19.1 \mathrm{mM} / \mathrm{cm}$ ) and cytochrome $c$ at 550-540 nm (extinction coefficient, $19.0 \mathrm{mM} / \mathrm{cm}$ ), respectively.

Quantitative polymerase chain reaction ( $q P C R)$ analysis. Total RNA was isolated using TRIzol reagent (Invitrogen Life Technologies). cDNA was synthesised with $1 \mu \mathrm{g}$ RNA and GoScript $^{\mathrm{TM}}$ reverse transcriptase (Promega, Madison, WI, USA) according to the manufacturer's instructions. qPCR assays were performed using power SYBR green PCR master mix and ABI 7500 Fast Real-Time PCR system (Applied Biosystems; Thermo Fisher Scientific, Inc., Waltham, MA, USA). The mRNA relative expression was calculated with the $2^{-\Delta \Delta \mathrm{Ct}}$ method (20). The primer sequences were as follows: PARP forward, 5'-GTGCCAACTACTGCCATACG-3' and 

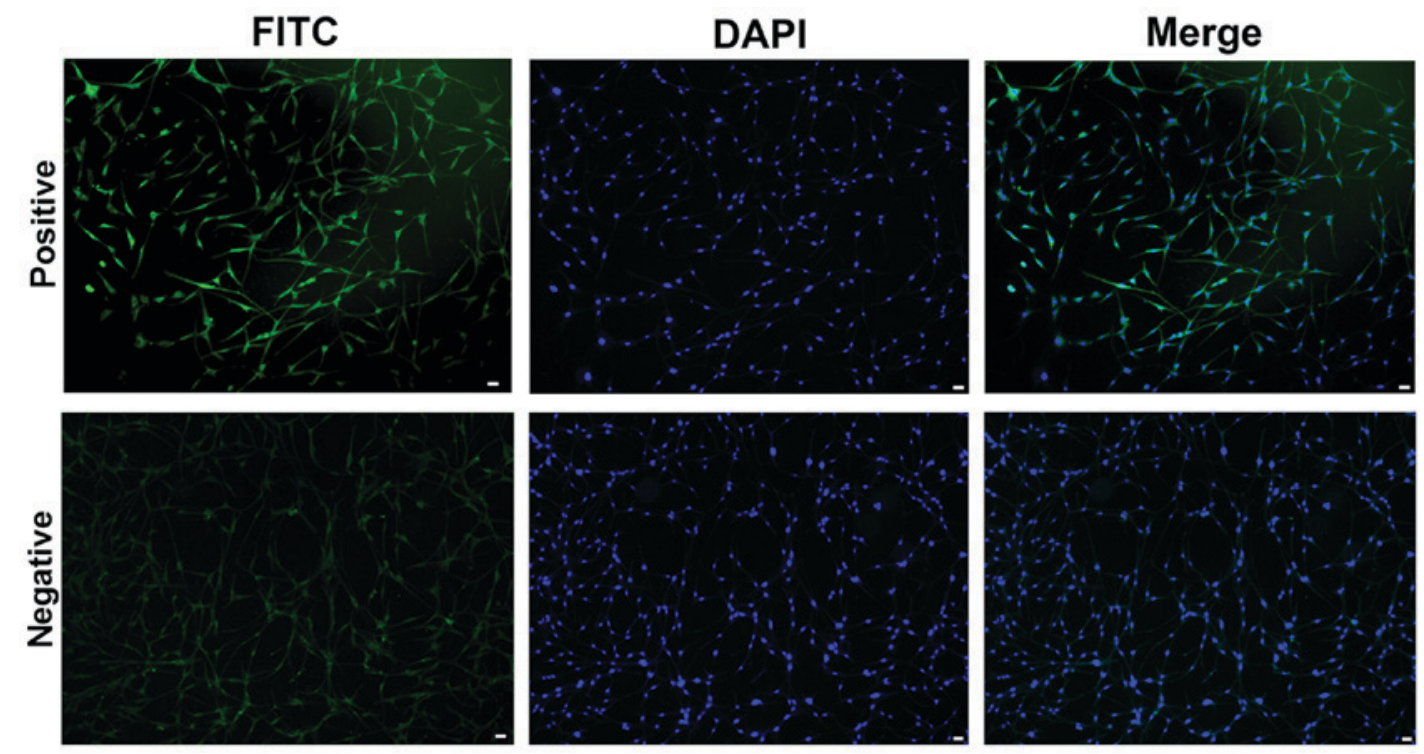

Figure 1. Identity of HUVECs with immunofluorescence staining. Scale bar, 200 um. HUVECs, human umbilical vein endothelial cells.

reverse, 5'-GCTATCATCAGACCCTCCCC-3'; caspase-3 forward, 5'-CATCGCTCTTGAAGACCAGC-3' and reverse, 5'-AGTCCAGTTCTGTACCACGG-3'; caspase-9 forward, 5'-AAGTGACCCTCCCAAGTAGC-3' and reverse, 5'-GTT CTGGCCAGGTCTCTTCT-3'; XIAP forward, 5'-TGTCCC TTTGATTACGGGCT-3' and reverse, 5'-AAGCCTGTAATC CCAGCACT-3'; GAPDH forward, 5'-CACAGTCCATGCCAT CACTG-3' and reverse, 5'-ATCTCGCTCCTGGAAGAT GG-3'.

Immunoblot analysis. Whole cell proteins were extracted using total protein extraction kit (Solarbio). Then, samples were separated by running them on sodium dodecyl sulfate-polyacrylamide gel electrophoresis (SDS-PAGE) before transferring to polyvinylidene fluoride (PVDF) membrane (Millipore, Billerica, MA, USA). After blocking non-specific antigen, the membrane was incubated at $4{ }^{\circ} \mathrm{C}$ overnight with primary antibodies for PARP, XIAP, caspase-3/-9, and GAPDH (Cell Signaling Technology, Inc., Danvers, MA, USA). The membrane was then incubated with horseradish peroxidase-conjugated goat anti-rabbit secondary antibody (Santa Cruz Biotechnology, Inc., Santa Cruz, CA, USA) for $1 \mathrm{~h}$. The blots were developed by enhanced chemiluminescence substrate (FD, China).

Statistical analysis. Data are presented as mean \pm standard deviation (SD) and analyzed by one-way analysis of variance (ANOVA) or t-test. P-value $<0.05$ was considered to indicate a statistically significant difference.

\section{Results}

Establishment of hyperglycemia injury model. First, the phenotype of HUVECs was identified using immunofluorescence staining (Fig. 1). Then, the CKK-8 detection showed that the cell viability of HUVECs declined gradually with an increase in the concentration of glucose. In contrast, cell viability was not affected by high mannitol content (Fig. 2). In

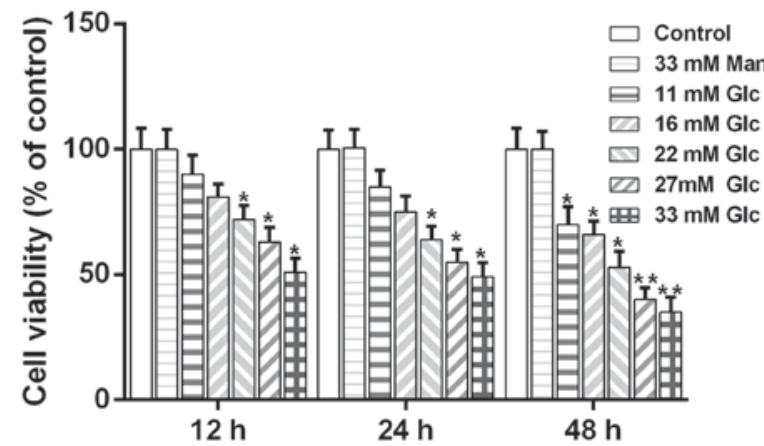

Figure 2. Cell viability detection by CKK-8 kit. Man, mannitol; Glu, glucose. Data were presented as mean $\pm \mathrm{SD}, \mathrm{n}=3$. ${ }^{*} \mathrm{P}<0.05$ and ${ }^{* *} \mathrm{P}<0.01$ vs. control.

this study, we employed $33 \mathrm{mM}$ glucose to stimulate HUVECs for establishment of the hyperglycemia injury model.

Obtusifolin protects HUVECs from oxidative stress. Oxidative stress is an important mediator of vascular complications in DM (21). The flow cytometry analysis displayed that cellular ROS was triggered under the high glucose environment. Conversely, the production of cellular ROS was reduced significantly after pretreatment with obtusifolin (Fig. 3A and B). Moreover, our results showed that the levels of MDA were significantly lower in the obtusifolin pretreatment groups than in the model group (Fig. 3C). Nevertheless, the activity detection assays displayed that both the CAT and SOD activities significantly increased in the obtusifolin pretreatment groups as compared to activities in the model group (Fig. 3D and E). Results further suggested that obtusifolin could protect HUVECs from oxidative stress in in a dose dependent manner.

Obtusifolin attenuates high glucose-induced mitochondrial dysfunction in HUVECs. According to results of the flow cytometry analysis, the production of mitochondrial ROS caused by hyperglycemia was obviously decreased by the pretreatment with obtusifolin, although there was no 

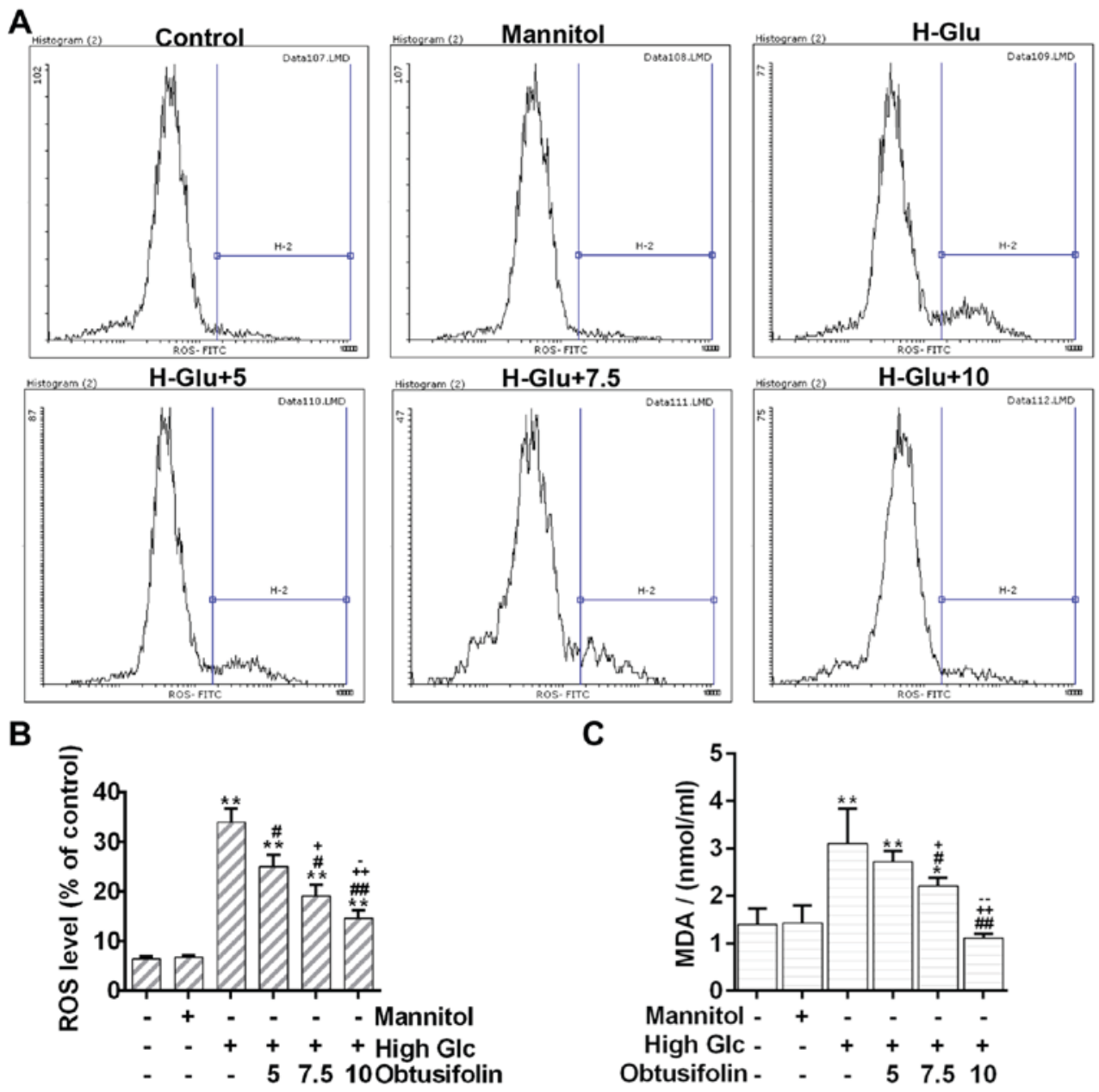

D

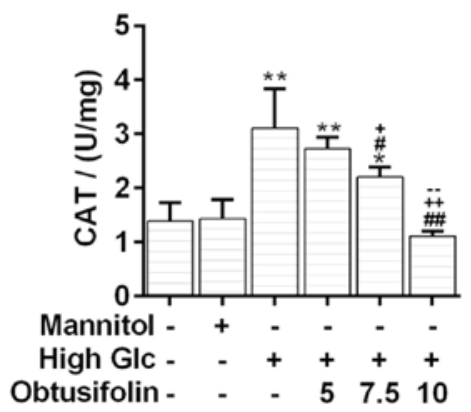

C

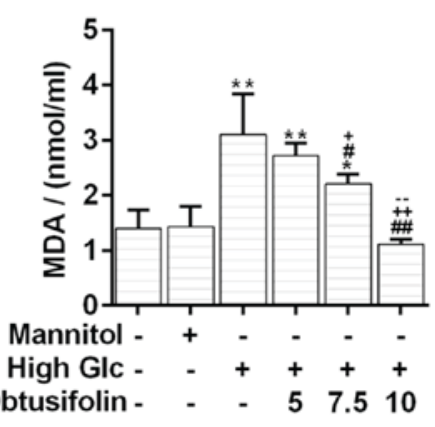

E

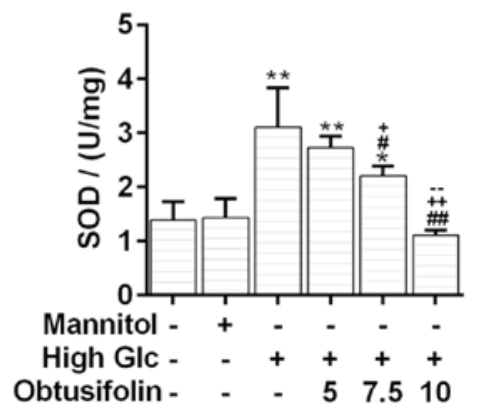

Figure 3. Obtusifolin protected HUVECs from oxidative stress. (A and B) Flow cytometry for cellular ROS analysis. (C) MDA levels. (D and E) Activity analysis of antioxidant enzyme CAT and SOD. Control group (control); high glucose model group (H-Glu); obtusifolin pretreatment groups (5, 7.5 and $10 \mathrm{mg} / \mathrm{ml}$ ): $\mathrm{H}-\mathrm{Glu}+5, \mathrm{H}-\mathrm{Glu}+7.5, \mathrm{H}-\mathrm{Glu}+10$. Data were presented as mean $\pm \mathrm{SD}, \mathrm{n}=4 .{ }^{*} \mathrm{P}<0.05$ and ${ }^{* *} \mathrm{P}<0.01$ vs. control; ${ }^{*} \mathrm{P}<0.05$ and ${ }^{\# \#} \mathrm{P}<0.01 \mathrm{vs}$. $\mathrm{H}-\mathrm{Glu}$; ${ }^{+} \mathrm{P}<0.05$ and ${ }^{++} \mathrm{P}<0.01$ vs. $\mathrm{H}-\mathrm{Glu}+5 ;-\mathrm{P}<0.05$ and ${ }^{-} \mathrm{P}<0.01$ vs. $\mathrm{H}-\mathrm{Glu}+7.5$. ROS, reactive oxygen species; MDA, malondialdehyde; CAT, catalase; SOD, superoxide dismutase.

significant difference between the medium concentration group and the low one (Fig. 4A and B). Moreover, the activities of mitochondrial respiratory chain complex I/III in the obtusifolin pretreatment groups were effectively recovered as compared to those in the model group (Fig. 4C). Furthermore, the MMP of HUVECs was recovered significantly after pretreatment with obtusifolin as compared to that in the model group (Fig. 5A and B). No significant difference was observed between the high concentration obtusifolin treatment group and the medium one with regard to the activity of mitochondrial respiratory chain complex III and the MMP. Nevertheless, the declined tendency of mitochondrial dysfunction in the obtusifolin pretreatment groups was still obvious.
Obtusifolin protects cells against high glucose-induced apoptosis. Mitochondrial dysfunction could activate mitochondria-dependent apoptosis pathway (22). The flow cytometry analysis showed that cell apoptosis caused by hyperglycemia was significantly inhibited in the obtusifolin pretreatment groups (Fig. 6A and B). In addition, our results showed that the expression of XIAP, an inhibitor of apoptosis proteins (23), was higher in the medium concentration btusifolin pretreatment group than in the model group according to the RT-PCR and western blot analyses. Meanwhile, the expression of PARP and caspase-3/-9, as pro-apoptotic proteins $(24,25)$, was significantly downregulated in the obtusifolin pretreatment groups as compared to in the model group (Fig. 7A-C). Conclusively, 
A
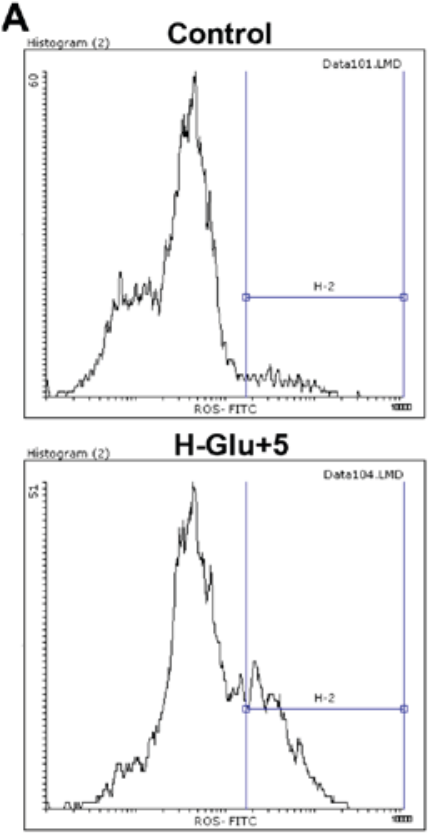

B

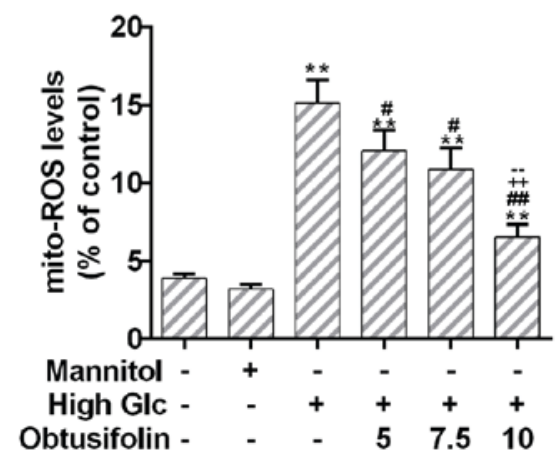

Mannitol
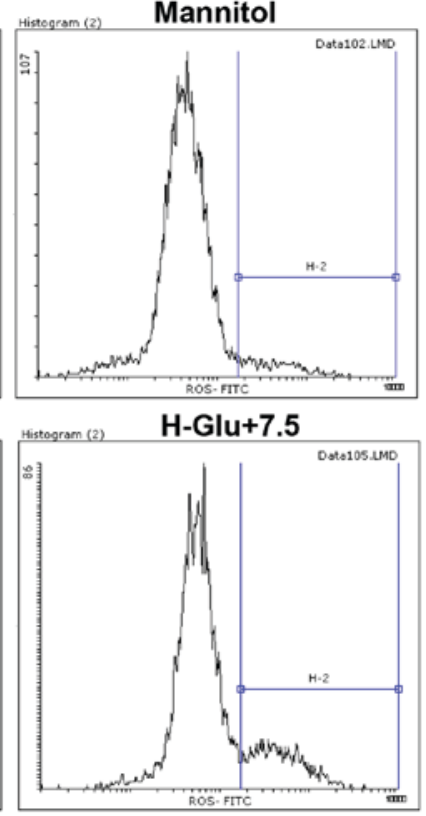

C

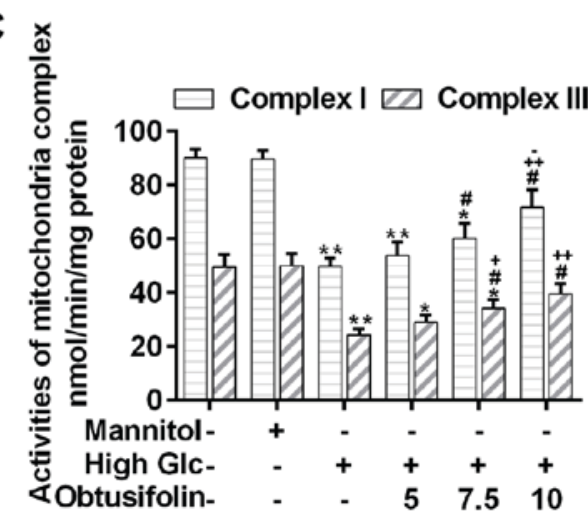

Figure 4. Obtusifolin decreased the generation of mitochondrial ROS. (A) Flow cytometry analysis for mitochondrial specific ROS. (B) Determination of mitochondrial specific ROS level. (C) Activity analysis of mitochondrial complex I/III. Control group (control); high glucose model group (H-Glu); obtusifolin pretreatment groups $(5,7.5$ and $10 \mathrm{mg} / \mathrm{ml})$ : H-Glu+5, H-Glu+7.5, H-Glu+10. Data were presented as mean $\pm \mathrm{SD}, \mathrm{n}=4$. ${ }^{*} \mathrm{P}<0.05$ and ${ }^{* *} \mathrm{P}<0.01 \mathrm{vs}$. control; ${ }^{\#} \mathrm{P}<0.05$ and ${ }^{\# \#} \mathrm{P}<0.01$ vs. $\mathrm{H}-\mathrm{Glu}$; ${ }^{+} \mathrm{P}<0.05$ and ${ }^{++} \mathrm{P}<0.01$ vs. $\mathrm{H}-\mathrm{Glu}+5$; ${ }^{-} \mathrm{P}<0.05$ and ${ }^{--} \mathrm{P}<0.01$ vs. H-Glu+7.5. ROS, reactive oxygen species.

it was suggested that obtusifolin protected HUVECs against high glucose-induced apoptosis.

Obtusifolin prevents the release of Omi/HtrA2 into the cytosol. $\mathrm{Omi} / \mathrm{HtrA} 2$ is released into the cytosol during apoptosis and promotes caspase pathway activation, which is a mitochondrial serine protease that resides in the mitochondria of healthy cells (14). Western blot analysis revealed that Omi/HtrA2 was released substantially into the cytosol under the high glucose condition. Interestingly, the release of Omi/HtrA2 was inhibited effectively in the obtusifolin pretreatment groups as compared to in the model group. Also, there was significant difference between the high concentration group and the low one (Fig. 8A and B).

\section{Discussion}

Macrovascular complications commonly occur in patients with diabetes mellitus, whose mortality accounts for more than $50 \%$ of all diabetes-related deaths (26). It has been reported that diabetes-associated hyperglycemia can induce apoptosis, which is a critical event in the onset and progression of diabetes mellitus $(27,28)$. Overproduction of ROS is a major cause of high glucose-induced apoptosis of endothelial cells (7). Moreover, oxidative stress could be attributed to the disruption of redox homeostasis (29). The bioactivity of obtusifolin has received much attention with regard to some physiological disorders (17). However, the effect of obtusifolin in the treatment of endothelial dysfunction and its possible molecular mechanisms still needs to be validated. Therefore, exploration of the potential effect of obtusifolin would provide new insights into its promising theoretical and translational value. In addition, it is possible that it will provide valuable reference for informed clinical application of other Chinese medicines as well.

Primary cells share great similarity with living cells in their genetic backgrounds. Moreover, HUVECs, as primary cells, originate from the fetal umbilical cord, which is not only abundant but also easy to obtain and work upon. These HUVECs have been used in the study of cardiovascular disease before $(30,31)$; thus, they were selected to set up an in vitro model in the present study. 


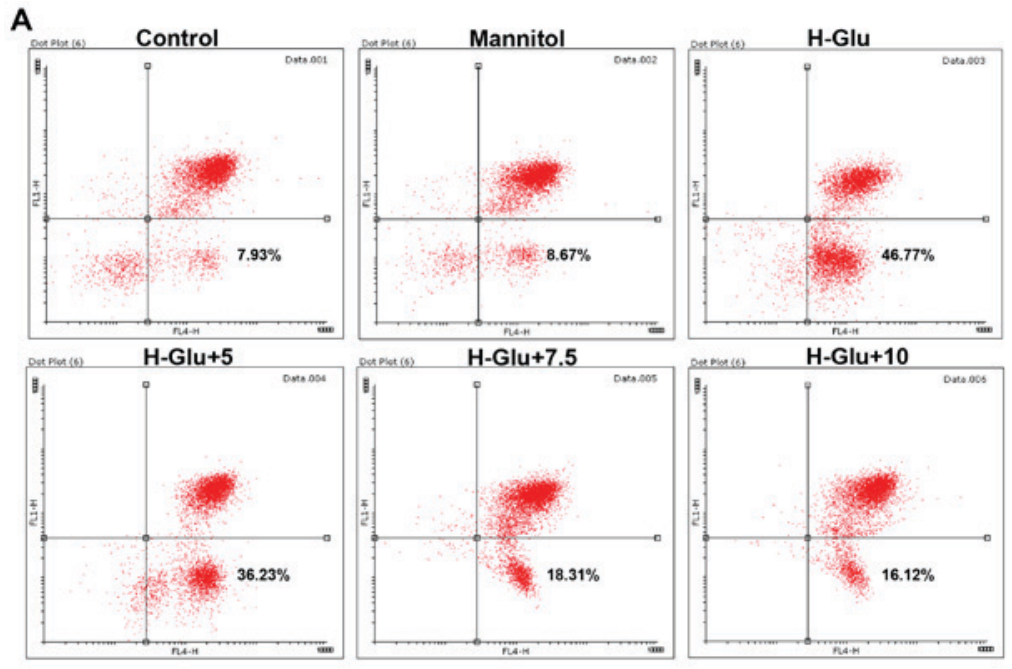

B

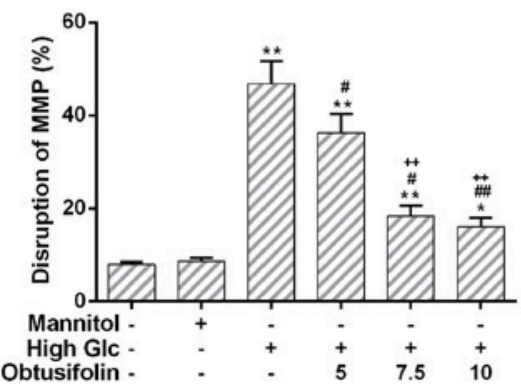

Figure 5. Obtusifolin recovered the loss of MMP. (A) Flow cytometry analysis for MMP. (B) The rate of the MMP disruption. Control group (control); high glucose model group (H-Glu); obtusifolin pretreatment groups $(5,7.5$ and $10 \mathrm{mg} / \mathrm{ml})$ : H-Glu+5, H-Glu+7.5, H-Glu+10. Data were presented as mean $\pm \mathrm{SD}, \mathrm{n}=4$. ${ }^{*} \mathrm{P}<0.05$ and ${ }^{* *} \mathrm{P}<0.01$ vs. control; ${ }^{*} \mathrm{P}<0.05$ and ${ }^{\# \#} \mathrm{P}<0.01$ vs. $\mathrm{H}-\mathrm{Glu} ;{ }^{+} \mathrm{P}<0.05$ and ${ }^{++} \mathrm{P}<0.01$ vs. H-Glu+5. MMP, mitochondrial membrane potential.

A
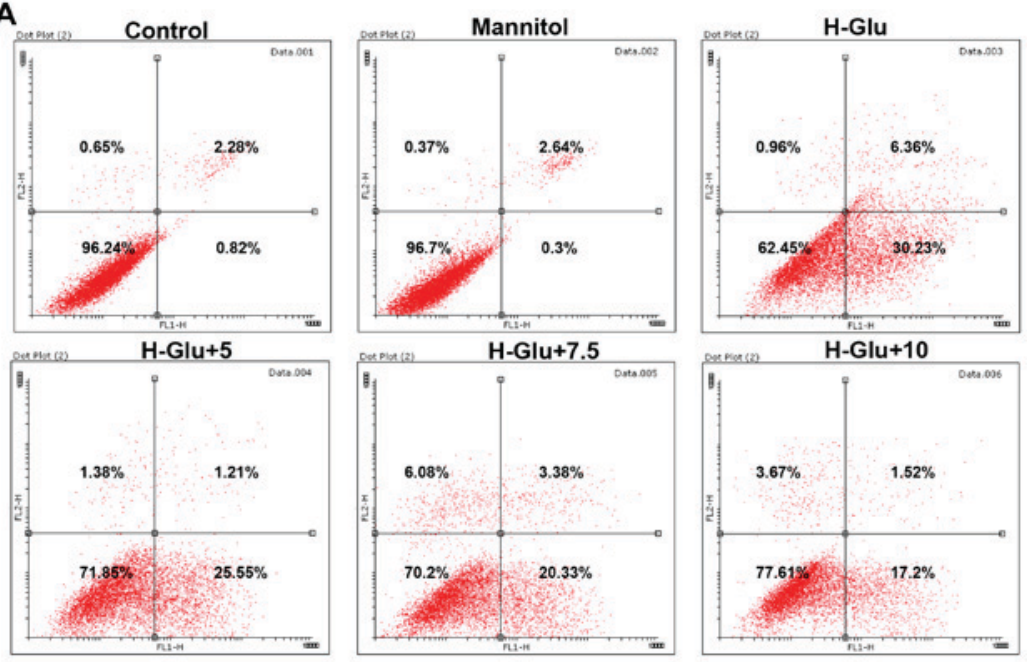

B

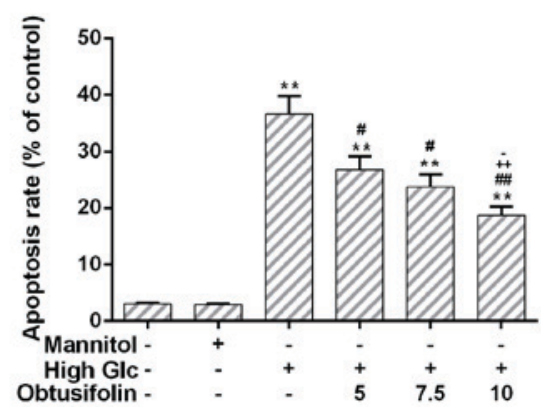

Figure 6. Obtusifolin protected against high glucose-induced apoptosis. (A) Flow cytometry analysis for apoptosis. (B) Determination of apoptosis rate. Control group (control); high glucose model group (H-Glu); obtusifolin pretreatment groups (5, 7.5 and $10 \mathrm{mg} / \mathrm{ml}$ ): H-Glu+5, H-Glu+7.5, H-Glu+10. Data were presented as mean $\pm \mathrm{SD}, \mathrm{n}=4 .{ }^{* *} \mathrm{P}<0.01$ vs. control; ${ }^{\#} \mathrm{P}<0.05$ and ${ }^{\# \#} \mathrm{P}<0.01$ vs. $\mathrm{H}-\mathrm{Glu} ;{ }^{++} \mathrm{P}<0.01$ vs. $\mathrm{H}-\mathrm{Glu}+5 ;{ }^{-} \mathrm{P}<0.05$ vs. H-Glu+7.5. 
A

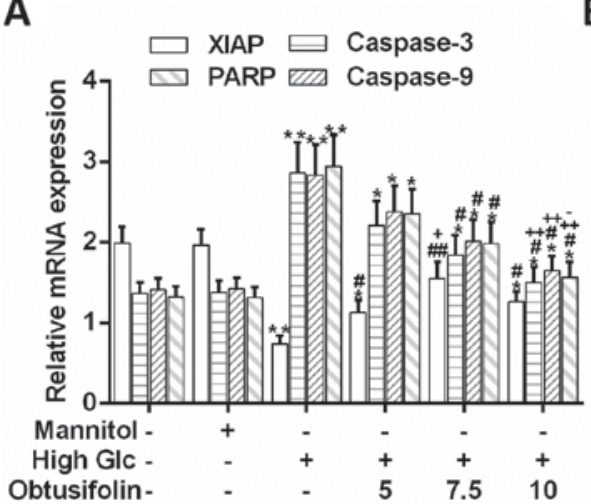

B

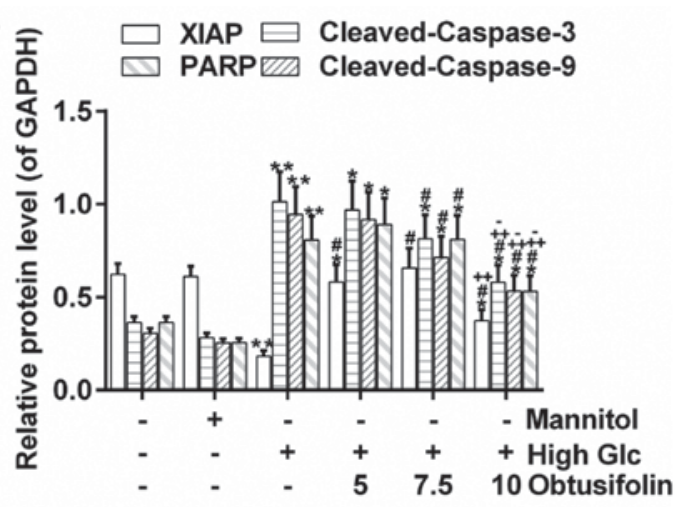

\section{C}

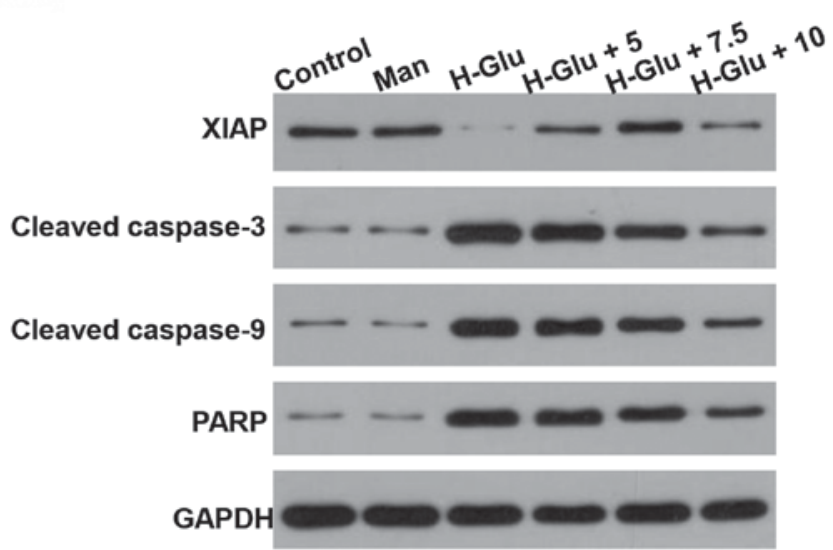

Figure 7. Obtusifolin inhibited the expression of apoptosis-related genes. (A) Quantitative analysis of PARP, caspase-3/-9 and XIAP. (B and C) Western blot analysis for XIAP, cleaved-caspase-3/-9 and PARP. GAPDH was assessed as sample loading control. Control group (control); mannitol (man); high glucose model group (H-Glu); obtusifolin pretreatment groups (5, 7.5 and $10 \mathrm{mg} / \mathrm{ml}$ ): $\mathrm{H}-\mathrm{Glu}+5, \mathrm{H}-\mathrm{Glu}+7.5, \mathrm{H}-\mathrm{Glu}+10$. Data were presented as mean $\pm \mathrm{SD}, \mathrm{n}=4$. ${ }^{*} \mathrm{P}<0.05$ and ${ }^{* *} \mathrm{P}<0.01$ vs. control; ${ }^{\#} \mathrm{P}<0.05$ and ${ }^{\# \#} \mathrm{P}<0.01$ vs. $\mathrm{H}-\mathrm{Glu} ;{ }^{+} \mathrm{P}<0.05$ and ${ }^{++} \mathrm{P}<0.01$ vs. H-Glu+5; $\mathrm{P}<0.05$ vs. H-Glu+7.5.
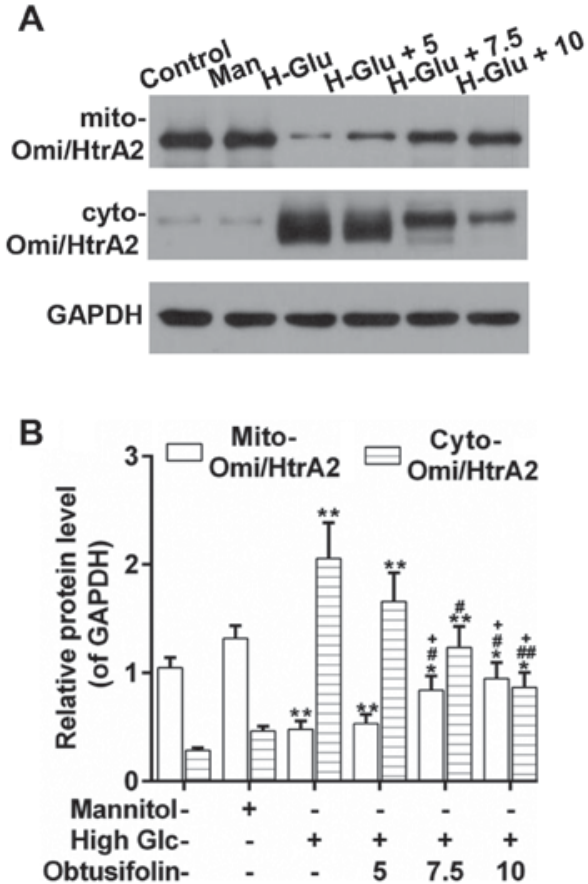

Figure 8. Obtusifolin prevented the release of Omi/HtrA2 into the cytosol. (A and B) Western blot analysis for the location of Omi/HtrA2. GAPDH was assessed as sample loading control. Control group (control); mannitol (man); high glucose model group (H-Glu); obtusifolin pretreatment groups (5, 7.5 and $10 \mathrm{mg} / \mathrm{ml}$ ): $\mathrm{H}-\mathrm{Glu}+5$, H-Glu+7.5, $\mathrm{H}-\mathrm{Glu}+10$. Data were presented as mean $\pm \mathrm{SD}, \mathrm{n}=4 .{ }^{*} \mathrm{P}<0.05$ and ${ }^{* * *} \mathrm{P}<0.01$ vs. control; ${ }^{*} \mathrm{P}<0.05$ and ${ }^{\# \#} \mathrm{P}<0.01$ vs. $\mathrm{H}-\mathrm{Glu} ;{ }^{+} \mathrm{P}<0.05$ and ${ }^{++} \mathrm{P}<0.01$ vs. $\mathrm{H}-\mathrm{Glu}+5$.

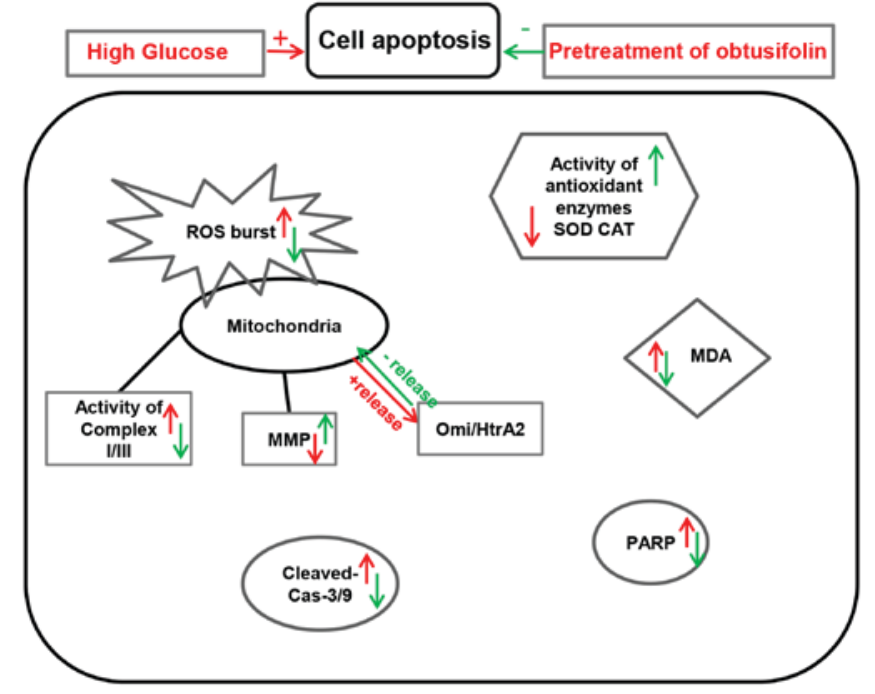

Figure 9. The work model of the protective role of obtusifolin in this study. The up arrow and the down arrow indicated the increase and decrease effect, respectively; the red one presented the function of high glucose, while the green one for the pretreatment of obtusifolin

In this investigation, cell viability was observed to decrease by glucose treatment in a dose-dependent manner. Hyperglycemia injury model was set up through high glucose treatment of HUVECs (Figs. 1 and 2). Our results showed that obtusifolin can decline the cellular ROS levels significantly 
(Fig. 3A and B). In addition, when accompanied by the relief of oxidative stress, the production of MDA-an end product of fatty acid peroxidation (32)-decreased in the obtusifolin pretreatment groups (Fig. 2C). Meanwhile, obtusifolin can help recover the activities of CAT and SOD (Fig. 2D), which are the main enzymes in the antioxidant defense system (33). Notably, obtusifolin can help maintain the balance between ROS-generation and ROS-removal in HUVECs. To investigate the origin of the increasing ROS generation and to evaluate if mitochondria are the main source of ROS, we determined the production of mitochondria-specific ROS by flow cytometry analysis. The results showed that mitochondria-specific ROS production was inhibited by the pretreatment with obtusifolin as compared to that in the model group (Fig. 4A and B). Furthermore, obtusifolin can increase the activities of mitochondrial respiratory chain complex I/III (Fig. 4C). The excessive ROS production is a critical step towards the opening of the mitochondrial membrane channel, which in turn, promotes the ROS production as well as oxidative stress, and thus, finally leads to apoptosis (34-36). Our results showed that obtusifolin could reverse the loss of the MMP (Fig. 5A and B). It was also implied by our results that obtusifolin plays an important role in the prevention of ROS generation and cellular oxidative stress. In addition, apoptosis induced by high glucose was inhibited in the obtusifolin pretreatment groups (Fig. 6A and B). Caspase-3/-9 have been recognized as apoptosis-related genes by extensive studies $(37,38)$. Caspase- 9 belongs to initiators of the cell apoptosis, which is able to cleave pro-caspase- 3 to active caspase-3 (also named cleaved caspase-3) (39). Upon its activation, caspase-3, which is a death protease frequently activated by extrinsic (death ligand) and intrinsic (mitochondrial) pathways, serves as the executor of apoptosis and then activates the apoptosis signals to downstream targets, including PARP $(37,38)$. By contrast, XIAP is considered to be an inhibitor of apoptosis proteins (23). In this study, the expression of XIAP was upregulated, whereas the expression of cleaved-PARP and cleaved-caspase-3/-9 was downregulated by the pretreatment with obtusifolin (Fig. 7A-C). One point that is worth consideration here is that the expression of XIAP was not higher in the high concentration obtusifolin pretreatment group than in the medium one. This can be explained by the fact that if the concentration of glucose is too high, treatment with obtusifolin may have toxic side effects on HUVECs, which may be due to the structure of anthraquinone in obtusifolin $(40,41)$. Additionally, we found that obtusifolin could prevent the release of Omi/HtrA2 into the cytosol effectively (Fig. 8A-C). These results suggested that obtusifolin protected HUVECs against high glucose-induced mitochondrial apoptosis mainly through the regulation of the location of $\mathrm{Omi} / \mathrm{HtrA} 2$.

It is known that Omi/HtrA2 can enhance caspase activity through multiple pathways (14). Thus, exploring the connections between obtusifolin and related signaling pathways would provide clues for the treatment of diabetes mellitus. Furthermore, whether obtusifolin can inhibit apoptosis through the caspase-independent pathway is worth investigating in detail in the future. Some studies have reported that endoplasmic reticulum stress pathway is involved in hyperglycemia-induced apoptosis (42); however, it has not been illustrated in this study. Besides, the protective effects of obtusifolin demonstrated in this study are largely from in vitro data. Thus, future explorations are needed to precisely define the effects of obtusifolin in vivo.

In conclusion, it was obvious that the protective effect of obtusifolin was enhanced with the increasing concentration. As shown in Fig. 9, obtusifolin protected HUVECs against high glucose-induced oxidative stress and mitochondrial dysfunction mainly through inhibition of ROS production and modulation of the activities of related enzymes. Meanwhile, obtusifolin could protect cells against high glucose-induced apoptosis in HUVECs, which was mainly due to aid in regulation of the release of Omi/HtrA2 into the cytosol. Based on these data, our study would provide effective therapeutic strategies in the treatment of DM.

\section{References}

1. Costantino S, Paneni F and Cosentino F: Hyperglycemia: A bad signature on the vascular system. Cardiovasc Diagn Ther 5: 403-406, 2015.

2. King GL, Shiba T, Feener EP and Nayak R: Cell culture model for the study of vascular complications of diabetes: The effect of high glucose levels on metabolism and growth of vascular cells. Hyperglyc Diab Vas Dis: 162-177, 1992.

3. Ceriello A, Quagliaro L, D'Amico M, Di Filippo C, Marfella R, Nappo F, Berrino L, Rossi F and Giugliano D: Acute hyperglycemia induces nitrotyrosine formation and apoptosis in perfused heart from rat. Diabetes 51: 1076-1082, 2002.

4. Baumgartner-Parzer SM, Wagner L, Pettermann M, Grillari J, Gessl A and Waldhäusl W: High-glucose-triggered apoptosis in cultured endothelial cells. Diabetes 44: 1323-1327, 1995.

5. Evans JL, Goldfine ID, Maddux BA and Grodsky GM: Oxidative stress and stress-activated signaling pathways: A unifying hypothesis of type 2 diabetes. Endocr Rev 23: 599-622, 2002.

6. Wang D, Wang Q, Yan G, Qiao Y, Sun L, Zhu B, Tang C and $\mathrm{Gu}$ Y: High glucose and interleukin $1 \beta$-induced apoptosis in human umbilical vein endothelial cells involves in down-regulation of monocarboxylate transporter 4. Biochem Biophys Res Commun 466: 607-614, 2015.

7. Zhao H, Ma T, Fan B, Yang L, Han C, Luo J and Kong L: Protective effect of trans- $\delta$-viniferin against high glucose-induced oxidative stress in human umbilical vein endothelial cells through the SIRT1 pathway. Free Radic Res 50: 68-83, 2016.

8. Du X, Stocklauser-Färber K and Rösen P: Generation of reactive oxygen intermediates, activation of NF-kappaB, and induction of apoptosis in human endothelial cells by glucose: Role of nitric oxide synthase? Free Radic Biol Med 27: 752-763, 1999.

9. Magalang UJ, Rajappan R, Hunter MG, Kutala VK, Kuppusamy P, Wewers MD, Marsh CB and Parinandi NL: Adiponectin inhibits superoxide generation by human neutrophils. Antioxid Redox Signal 8: 2179-2186, 2006.

10. Surico D, Farruggio S, Marotta P, Raina G, Mary D, Surico N, Vacca $G$ and Grossini E: Human chorionic gonadotropin protects vascular endothelial cells from oxidative stress by apoptosis inhibition, cell survival signalling activation and mitochondrial function protection. Cell Physiol Biochem 36: 2108-2120, 2015.

11. Chen W, Zheng G, Yang S, Ping W, Fu X, Zhang N, Wang DW and Wang J: CYP2J2 and EETs protect against oxidative stress and apoptosis in vivo and in vitro following lung ischemia/reperfusion. Cell Physiol Biochem 33: 1663-1680, 2014.

12. Prudent $\mathrm{J}$ and McBride HM: Mitochondrial dynamics: ER actin tightens the Drp1 noose. Curr Biol 26: R207-R209, 2016.

13. Vaux DL and Silke J: HtrA2/Omi, a sheep in wolf's clothing. Cell 115: 251-253, 2003.

14. Suzuki Y, Takahashi-Niki K, Akagi T, Hashikawa T and Takahashi R: Mitochondrial protease Omi/HtrA2 enhances caspase activation through multiple pathways. Cell Death Differ 11: 208-216, 2004.

15. Ju MS, Kim HG, Choi JG, Ryu JH, Hur J, Kim YJ and Oh MS: Cassiae semen, a seed of Cassia obtusifolia, has neuroprotective effects in Parkinson's disease models. Food Chem Toxicol 48: 2037-2044, 2010.

16. Patil UK, Saraf S and Dixit VK: Hypolipidemic activity of seeds of Cassia tora Linn. J Ethnopharmacol 90: 249-252, 2004. 
17. Tang $\mathrm{Y}$ and Zhong $\mathrm{Z}$ : Obtusifolin treatment improves hyperlipidemia and hyperglycemia: Possible mechanism involving oxidative stress. Cell Biochem Biophys 70: 1751-1757, 2014.

18. Ding F, Shao ZW, Yang SH, Wu Q, Gao F and Xiong LM: Role of mitochondrial pathway in compression-induced apoptosis of nucleus pulposus cells. Apoptosis 17: 579-590, 2012.

19. Frazier AE and Thorburn DR: Biochemical analyses of the electron transport chain complexes by spectrophotometry. Methods Mol Biol 837: 49-62, 2012

20. Livak KJ and Schmittgen TD: Analysis of relative gene expression data using real-time quantitative PCR and the 2(-Delta Delta C(T)) method. Methods 25: 402-408, 2001.

21. Ha $\mathrm{H}$ and Lee HB: Reactive oxygen species as glucose signaling molecules in mesangial cells cultured under high glucose. Kidney Int Suppl 77: S19-S25, 2000.

22. Soon BH, Abdul Murad NA, Then SM, Abu Bakar A, Fadzil F, Thanabalan J, Mohd Haspani MS, Toh CJ, Mohd Tamil A, Harun R, et al: Mitochondrial DNA mutations in grade II and III glioma cell lines are associated with significant mitochondrial dysfunction and higher oxidative stress. Front Physiol 8: 231, 2017.

23. Rajcan-Separovic E, Liston P, Lefebvre C and Korneluk RG: Assignment of human inhibitor of apoptosis protein (IAP) genes xiap, hiap-1 and hiap-2 to chromosomes Xq25 and 11q22-q23 by fluorescence in situ hybridization. Genomics 37: 404-406, 1996.

24. Boulares AH, Zoltoski AJ, Yakovlev A, Xu M and Smulson ME: Roles of DNA fragmentation factor and poly (ADP-ribose) polymerase in an amplification phase of tumor necrosis factor-induced apoptosis. J Biol Chem 276: 38185-38192, 2001.

25. Julien O and Wells JA: Caspases and their substrates. Cell Death Differ 24: 1380-1389, 2017.

26. Stehouwer CD: Vascular complications in diabetes mellitus: Role of endothelial dysfunction. Ned Tijdschr Geneeskd 140: 870-874, 1996 (In Dutch).

27. Altabas V: Diabetes, endothelial dysfunction and vascular repair: What should a diabetologist keep his eye on? Int J Endocrinol 2015: 848272, 2015.

28. Caballero AE: Endothelial dysfunction in obesity and insulin resistance: A road to diabetes and heart disease. Obes Res 11: 1278-1289, 2003.

29. Baynes JW: Role of oxidative stress in development of complications in diabetes. Diabetes 40: 405-412, 1991.

30. Agnoletti L, Bachetti T, Bastianon D, Francolini G and Ferrari R Rosuvastatin stimulates eNOS and inhibits apoptosis in HUVECs exposed to sera from cardiovascular diseases patients. J Mol Cell Cardio 42 (Suppl): S226, 2007.

31. Udvardy M, Posan E, Harsfalvi J, Kaplar M, Batar P and Altorjay I: 88. In vitro clot-lysis in the presence of cultured human umbilical vein endothelial cells, experiences in diabetes mellitus and liver cirrhosis. Fibrinolysis 10 (Suppl 1): S27, 1996.
32. Tsikas D: Assessment of lipid peroxidation by measuring malondialdehyde (MDA) and relatives in biological samples: Analytical and biological challenges. Anal Biochem 524: 13-30, 2017.

33. Sharafati-Chaleshtori R, Shirzad H, Rafieian-Kopaei M and Soltani A: Melatonin and human mitochondrial diseases. J Res Med Sci 22: 2, 2017.

34. Zorov DB, Filburn CR, Klotz LO, Zweier JL and Sollott SJ: Reactive oxygen species (ROS)-induced ROS release: A new phenomenon accompanying induction of the mitochondrial permeability transition in cardiac myocytes. J Exp Med 192: 1001-1014, 2000.

35. Barateiro A, Vaz AR, Silva SL, Fernandes A and Brites D: ER stress, mitochondrial dysfunction and calpain/JNK activation are involved in oligodendrocyte precursor cell death by unconjugated bilirubin. Neuromolecular Med 14: 285-302, 2012.

36. Vincent AM, Russell JW, Low P and Feldman EL: Oxidative stress in the pathogenesis of diabetic neuropathy. Endocr Rev 25: 612-628, 2004

37. Chai WS, Zhu XM, Li SH, Fan JX and Chen BY: Role of Bcl-2 family members in caspase-3/9-dependent apoptosis during Pseudomonas aeruginosa infection in U937 cells. Apoptosis 13: 833-843, 2008

38. Seol JG, Park WH, Kim ES, Jung CW, Hyun JM, Lee YY and Kim BK: Potential role of caspase-3 and -9 in arsenic trioxide-mediated apoptosis in PCI-1 head and neck cancer cells. Int J Oncol 18: 249-255, 2001.

39. Yin Q, Park HH, Chung JY, Lin SC, Lo YC, da Graca LS, Jiang $\mathrm{X}$ and $\mathrm{Wu} \mathrm{H}$ : Caspase-9 holoenzyme is a specific and optimal procaspase-3 processing machine. Mol Cell 22: 259-268, 2006.

40. Xue W and Warshawsky D: Metabolic activation of polycyclic and heterocyclic aromatic hydrocarbons and DNA damage: A review. Toxicol Appl Pharmacol 206: 73-93, 2005.

41. Haruna K, Kanezaki H, Tanabe K, Dai WM and Nishimoto S: Effects of structural modification on the DNA binding properties and photo-induced cleavage reactivity of propargylic sulfones conjugated with an anthraquinone structure. Bioorg Med Chem 14: 4427-4432, 2006.

42. Gwak H, Kim S, Dhanasekaran DN and Song YS: Resveratrol triggers ER stress-mediated apoptosis by disrupting N-linked glycosylation of proteins in ovarian cancer cells. Cancer Lett 371: 347-353, 2016. 